\title{
Forum
}

\section{Dossier Interdisciplinarité Invoquées, pratiquées, critiquées : les interdisciplinarités en débat}

Le comité de rédaction de Natures Sciences Sociétés souhaite ouvrir avec ce numéro 1 de l'année 2004 un dossier sur l'état des pratiques interdisciplinaires dans les communautés de scientifiques et de praticiens en prise avec le traitement de problèmes complexes.

Ce dossier prendra la forme d'un débat, sur la base de textes d'ordres très divers : témoignages, points de vue personnels, notes de lecture, réflexions théoriques aussi bien qu'expériences pratiques, etc. Il s'agit de faire le point sur les pratiques interdisciplinaires dans les grands établissements de recherche et universitaires français ou étrangers, mais également dans des dispositifs plus modestes ou dans des structures plus éloignées des canons de la recherche publique. Au-delà des échanges et des controverses, que nous souhaitons voir s'ouvrir, nous visons à faire progresser le débat d'idées sur l'interdisciplinarité, si souvent prônée par les établissements de recherche comme dans la plupart des appels d'offre, plus ou moins «bricolée » au sein des laboratoires et des équipes, mais encore bien peu reconnue!

La revue a été créée pour être un lieu de débats pour les travaux interdisciplinaires entre sciences de la nature, sciences techniques et sciences de la société. Les thématiques liées aux ressources naturelles ont souvent été et demeurent - des points d'application privilégiés de ces démarches, en associant, en particulier, écologie, hydrologie, agronomie, zootechnie, économie, sociologie, géographie, anthropologie. Mais, bien souvent par l'intermédiaire ou sous l'influence de la question de l'environnement, ce genre d'interdisciplinarité s'est développé dans d'autres domaines de recherche (comme l'eau, la ville, la santé, l'alimentation, les biotechnologies, etc.), en se cristallisant notamment autour des notions de risque et de développement durable et en impliquant ainsi de nouveaux domaines disciplinaires. C'est bien cette interdisciplinarité large et ambitieuse, quel qu'en soit le thème, qui est au cœur de notre propos.
La complexité des problèmes ainsi soulevés rend nécessaire la constitution de dispositifs de recherche rigoureux, associant des disciplines complémentaires dès la phase de conception des problématiques et de leur déclinaison en cadres théoriques et en démarches méthodologiques. Ainsi conduite, si ce n'est contrainte, à expliciter - et donc à énoncer - ses concepts et ses méthodes, et à mettre en valeur ses résultats, l'interdisciplinarité s'est consolidée. Elle a, en outre, montré sa pertinence à de nombreuses occasions pour traiter des questions considérées comme complexes. Ce fut - et cela demeure l'objectif de Natures Sciences Sociétés que de contribuer par l'évaluation et la publication scientifiques à cette consolidation et à cette valorisation. Après dix années d'existence, le temps nous paraît venu de faire le point sur les acquis en la matière dans la communauté scientifique française.

Qu'en est-il d'abord dans les institutions et les établissements de recherche? En effet, les principaux établissements de recherche français paraissent parfois mettre leurs agents dans des injonctions paradoxales en les incitant officiellement à la transversalité et à l'interdisciplinarité... sans pour autant changer les cultures disciplinaires (ce qui ne se fait effectivement pas par décision bureaucratique), ni les critères d'évaluation qui sont utilisés pour les recrutements et les promotions des chercheurs, qui restent la plupart du temps fondés sur l'excellence disciplinaire. On pourrait raisonnablement attendre sur ce second point que les établissements incitent à l'identification de critères complémentaires, plus cohérents avec leur demande d'interdisciplinarité, qu'ils justifient eux-mêmes par la pertinence qu'ils attendent de travaux de recherches en phase avec les questions de société qui leurs sont soumises par leurs tutelles et par le monde social et politique. L'alignement habituel sur les critères académiques, souvent présenté comme une norme internationale tirée par le système de recherche 
anglo-saxon, est un faux-fuyant visant à éluder la question, car s'il en est qui savent prendre en compte dans leurs évaluations la pertinence sociale, l'originalité des recherches et leur contribution à l'histoire des idées, ce sont bien les Anglo-Saxons!

Mais l'interdisciplinarité ne se cantonne pas au monde de l'Université et des grands établissements de recherche, elle touche les grandes écoles d'ingénieurs, ainsi que des associations regroupant public et privé autour de questions conceptuelles, méthodologiques et... pratiques : elle imprègne de plus en plus la pensée et la pratique de nombreux décideurs, personnel politique, dirigeants d'entreprises, enseignants, ingénieurs, dans des domaines divers; elle est attendue, par exemple, dans la plupart des grands programmes internationaux à l'initiative de la Commission européenne, des agences de l'ONU, de l'OCDE ou du ministère des Affaires étrangères. Elle se justifie par la complexité des questions soulevées et des objets conçus pour les traiter. Elle se justifie souvent également par l'exigence d'opérationnalité exprimée par des praticiens ayant à agir dans des situations jugées complexes, marquées par l'incertitude et l'incomplétude des informations disponibles. Il n'est pas concevable d'ignorer aujourd'hui toutes ces expériences qui vont dans le même sens, quels qu'en soient les domaines d'application, les référents et les conceptions quant aux modalités d'acquisition des connaissances et aux rapports de celles-ci avec l'action. Nous souhaitons développer des relations avec les groupes qui sont à leur origine. C'est pourquoi le comité de rédaction ouvre un dossier qu'il souhaite voir se prolonger sur plusieurs numéros, les premiers textes suscitant réponses et réactions de nos lecteurs, ainsi que d'autres initiatives provenant de groupes et de cercles plus éloignés.

La revue a bien sûr déjà publié de nombreux textes qui abordent toutes ces questions. Mais, pour ouvrir ce dossier destiné à stimuler et élargir le débat, nous commençons dans ce numéro avec quatre textes qui illustrent - sans l'épuiser - la diversité des approches possibles : Rémi Barré fait part de sa lecture de l'ouvrage de Helga Nowotny et al., Re-thinking Science : Knowledge and the Public in an Age of Uncertainty; cet ouvrage fait suite à celui de Gibbons et al., The New Production of Knowledge, qui avait présenté le concept de «recherche de mode 2 ». Il nous éclaire ainsi sur les théorisations en cours, dans le monde anglo-saxon, de pratiques de recherche proches de celles que plusieurs d'entre nous mettent également en œuvre. Pour amorcer le débat sur l'interdisciplinarité dans les institutions de recherche, Daniel Terrasson et Pierrick Givone analysent l'état de la pratique et du discours sur l'interdisciplinarité au Cemagref, puis Daniel Bley évoque la recomposition laborieuse des sections du département des Sciences de l'homme et de la société du CNRS. Enfin, Jean-Marie Legay rend compte des débats qui ont eu lieu à l'occasion des Journées de l'association NSS-Dialogues de décembre 2002, en donnant la parole aux participants sur leurs pratiques interdisciplinaires dans les recherches sur le vivant.

Nous souhaitons que ces quatre prises de position entraînent des réactions et que nous soient proposés d'autres regards, qu'ils convergent ou divergent avec ces premières positions. Les controverses sont inhérentes au débat scientifique et l'interdisciplinarité n'existera que si elle prend corps dans un certain nombre de controverses partagées par toute une communauté scientifique. C'est pourquoi nous souhaitons le débat le plus large possible impliquant tous ceux qui se sentent concernés par la nécessité de confronter les domaines disciplinaires et de créer des synergies entre eux.

Le comité de rédaction

To access this journal online: www.edpsciences.org 